OPEN ACCESS

Edited by:

Georgia Konstantinidou, University of Bern, Switzerland

Reviewed by: Ignacio Varela, University of Cantabria, Spain Piera Tocci,

Regina Elena National Cancer Institute (IRCCS), Italy

*Correspondence: Yan Zhang

Chzhangyan@outlook.com

Specialty section: This article was submitted to Molecular and Cellular Oncology, a section of the journa

Frontiers in Oncology

Received: 09 August 2020 Accepted: 21 December 2020 Published: 03 February 2021

Citation:

Liu J, Xu T, Jin Y, Huang B and Zhang $Y$ (2021) Progress and Clinical

Application of Single-Cell

Transcriptional Sequencing Technology in Cancer Research.

Front. Oncol. 10:593085. doi: 10.3389/fonc.2020.593085

\section{Progress and Clinical Application of Single-Cell Transcriptional Sequencing Technology in Cancer Research}

\author{
Jian Liu ${ }^{1}$, Tianmin $X u^{1}$, Yuemei Jin ${ }^{1}$, Bingyu Huang ${ }^{1}$ and Yan Zhang ${ }^{2 *}$ \\ ${ }^{1}$ Department of Gynaecology and Obstetrics, Jilin University Second Hospital, ChangChun, China, ${ }^{2}$ Department of Breast \\ Surgery, Jilin University Second Hospital, ChangChun, China
}

Cancer has been a daunting challenge for human beings because of its clonal heterogeneity and compositional complexity. Tumors are composed of cancer cells and a variety of non-cancer cells, which together with the extracellular matrix form the tumor microenvironment. These cancer-related cells and components and immune mechanisms can affect the development and progression of cancer and are associated with patient diagnosis, treatment and prognosis. As the first choice for the study of complex biological systems, single-cell transcriptional sequencing (scRNA-seq) has been widely used in cancer research. ScRNA-seq has made breakthrough discoveries in tumor heterogeneity, tumor evolution, metastasis and spread, development of chemoresistance, and the relationship between the tumor microenvironment and the immune system. These results will guide clinical cancer treatment and promote personalized and highly accurate cancer treatment. In this paper, we summarize the latest research progress of scRNA-seq and its guiding significance for clinical treatment.

Keywords: scRNA-seq, precision medicine, tumor heterogeneity, tumor microenvironment, cancer cells

\section{INTRODUCTION}

The expression measured by traditional bulk transcriptome sequencing (Bulk RNA-seq) is the average expression of hundreds of single cells in the sample, so it is difficult to get the heterogeneity information of the cell population, and those cells that deviate from the average expression level may have important biological significance. Among the many genomic technologies currently available, scRNA-seq is the most useful and reliable method for detecting the biological mechanisms of lowexpressing cell populations in tumors (1). Transcriptome sequencing based on the expression level of individual cells has also greatly expanded the application scope of transcriptome research.

Since the single-cell transcriptomic analysis was first reported (2), many other scRNA-seq methods have been developed, such as MARS-seq (Massively parallel RNA single cell sequencing), Smart-seq(Switching mechanism at $5^{\prime}$ end of the RNA template sequencing), Cel-seq(Cell expression by linear amplification and sequencing, MATQ-seq (multiple annealing and dCtailing-based quantitative single-cell RNA-seq), etc. The difference between them is mainly the method to amplify the mRNA transcript to produce a full-length cDNA with a unique molecular identity (UMI) at the $5^{\prime}$ or $3^{\prime}$ end. In order to promote automation and simplify sample preparation, 
droplet-based ultra-high-throughput single-cell sequencing technology relying on cellular bar coding has emerged since 2015. It is divided into the inDrop (3) and Drop-seq (4) systems developed by David Weitz et al. at Harvard University and the Chromium platform of 10x Genomics Company (5). Based on the new Chromium system of 10x Genomics, thousands or even tens of thousands of single-cell population analyzes can be achieved, thus overcoming the shortcomings of conventional scRNA-seq methods in terms of throughput or scalability, and providing new ideas for single-cell studies (6).

General scRNA-seq workflow includes many steps (Figure 1). All single-cell protocols start with a suspension of cells. For most tissues, this means that beforehand, the extracellular matrix that holds cells together has to be processed to loosen this mesh and to induce the release of cells into suspension. In a lot of cases, dissociation protocols are combined of both approaches by cutting tissues into small pieces, which are then dissociated by an enzymatic treatment. The principle of droplet-based ultrahigh-throughput single-cell sequencing technology is built on the microfluidic control system to produce nanoscale droplets of single cells and single microspheres. Each microsphere surface is wrapped with a specific oligonucleotide sequence (cellular barcode) and UMIs to mark the RNA molecules from the same cell (7). Next, cells dissolve in the reaction system and undergo reverse transcription to form full-length cDNA sequences. The minute amounts of cDNA are then amplified by PCR to construct a cDNA library and the Illumina platform are used to sequence it after qualifying (8). In a subsequent step, we use a series of computing tools to process, analyze and visualize these datasets in order to obtain heterogeneity information (9).

ScRNA-seq has been applied in tumor research, such as dividing tumors into different subtypes to explore cell development trajectory (10), constructing a microenvironmental blueprint for tumors (11), discovering new biomarkers and monitoring circulating tumor cells (12), discovering rare cells and mapping them (13), identifying the mechanism of drug resistance and finding new therapeutic targets (14), explaining paracrine signaling networks (15), and making a strategy to develop combined therapy to target multiple related cells in the tumor. ScRNA-seq has been used in a variety of cancer studies (Table 1). In this paper, we will present the latest results from cancer studies using 10x Genomics scRNA-seq.

\section{TUMOR CELL HETEROGENEITY STUDY}

Different types of tumors, the same tumor formed by different individuals, and even cancer cells of the same clone are usually heterogeneous, and this heterogeneity can change as the disease progresses (37). ScRNA-seq allows the study of alternative polyadenylation (APA) patterns and gene expression levels of tumor cells in different tumors. In addition, APA and gene expression in specific cell types are consistent, suggesting that cell

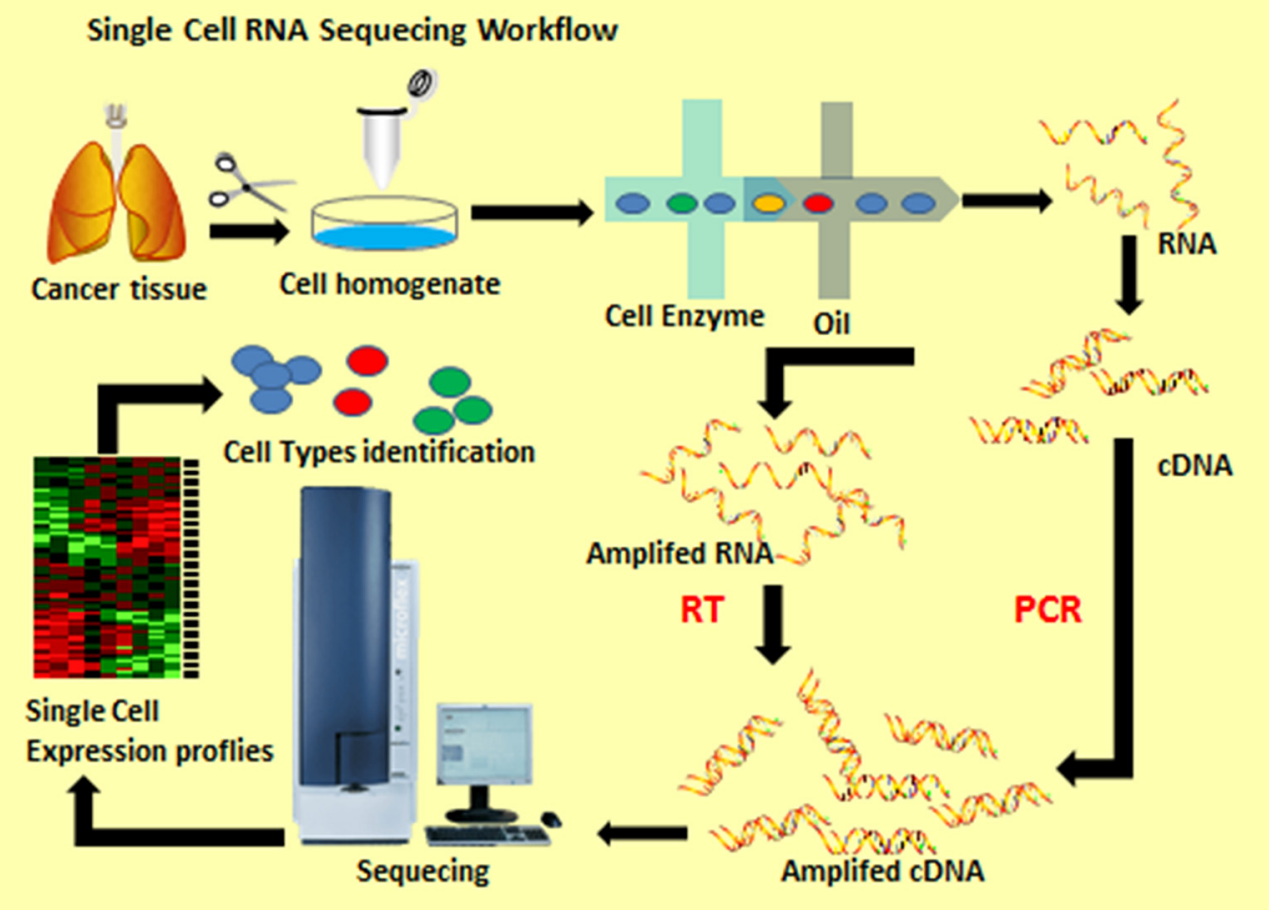

FIGURE 1 | Single cell RNA sequencing workflow. 
TABLE 1 | Summary of the studies in human tumors using scRNAseq.

\begin{tabular}{|c|c|c|c|c|}
\hline Cancer type & Tumor cell types & scRNAseq method & Cell number & References \\
\hline Follicular B-cell lymphomas & Cancer tissue cells & $10 \times$ Genomics & 34,188 & $(16)$ \\
\hline Breast cancer (TNBC) & CD3+ TILs & $\begin{array}{l}\text { 10×Genomics and Fluidigm } \\
\text { C1 }\end{array}$ & 6,311 & $(17)$ \\
\hline Breast cancer (TNBC) & PDX & $10 \times$ Genomics & 3,500 & $(18)$ \\
\hline Breast cancer (TNBC) & M6-Ctrl,M6-Hh cells & $10 \times$ Genomics & 14,950 & $(19)$ \\
\hline Breast cancer & Cancer epithelial cells & $\begin{array}{l}10 \times \text { Genomics and Fluidigm } \\
\text { C1 }\end{array}$ & 24,646 & (20) \\
\hline Gliomas & Inter $\left(\mathrm{CD} 11 \mathrm{~b}^{+}\right)$and Intra tumor TAMs (in silico) & $\begin{array}{l}\text { 10×Genomics and Fluidigm } \\
\text { C1 }\end{array}$ & 5,455 & $(21)$ \\
\hline $\begin{array}{l}\text { Gastric Premalignant Lesions and Early Gastric } \\
\text { Cancer }\end{array}$ & Cancer epithelial cells & $10 \times$ Genomics & 56,440 & $(22)$ \\
\hline Melanoma & PDX & $10 \times$ Genomics & 8,700 & (23) \\
\hline Melanoma & 451Lu-Par, 451Lu-BR, A375-BR,451Lu-BR3 & $10 \times$ Genomics & 6,500 & $(24)$ \\
\hline Merkel cell carcinoma (MCC) & cells & 10×Genomics 3' Chromium & 11,021 & (25) \\
\hline Leukemia & Cancer tissue cells & $10 \times$ Genomics & 5,000 & (26) \\
\hline Human kidney tumors & CMVpp65- or PRAME-specific T cells & $10 \times$ Genomics & 72,501 & $(27)$ \\
\hline Hepatocellular carcinoma & normal and cancerous kidney cells & $10 \times$ Genomics & 7,104 & (28) \\
\hline$(\mathrm{HCC})$ & circulating tumor cells (CTCs) & 10x Genomics & 3,847 & (29) \\
\hline Hepatocellular carcinoma & $\mathrm{HuH} 1$ and $\mathrm{HuH} 7$ cell lines & $10 \times$ Genomics & 5,063 & (30) \\
\hline Hepatocellular carcinoma & T cells & $10 \times$ Genomics & 57,530 & (31) \\
\hline Pancreatic ductal adenocarcinoma & pancreatic cells & $10 \times$ Genomics & 16,128 & (32) \\
\hline Human glioblastomas & EGFR wild-type and EGFRvIll mutant cells & & & \\
\hline Non-small-cell lung cancer (NSCLC) & stromal cells & $10 \times$ Genomics & 84,381 & (33) \\
\hline T-cell acute lymphoblastic leukemia (T-ALL) & T-ALL cells & $10 \times$ Genomics & 2,074 & (34) \\
\hline Alveolar Rhabdomyosarcoma (ARMS) & circulating tumor cells & $10 \times$ Genomics & 416 & (35) \\
\hline Head and neck squamous cell carcinoma (HNSCC) & Cancer tissue cells & $10 \times$ Genomics & 5,902 & (36) \\
\hline
\end{tabular}

types can be identified based on changes in the length of 3 'untranslated region (3' UTR) combined with gene expression (38).

\section{Heterogeneity Study of Subtypes of Tumor Cells}

In one study, scRNA-seq was used to identify two catheter subtypes with abnormal and malignant gene expression profiles, respectively, from primary PDAC tumors, and the malignant subtype consisted of several subgroups with different proliferation and migration potentials (31). Interestingly, scRNA-seq indicated that there were three different groups of mammary epithelial cells, and that multiple subclusters were present in each major epithelial cell type (20). At the single-cell level, researchers also found liver CSCs and demonstrated that liver CSCs are heterogeneous in phenotype, function and transcriptome, and that different genes in different CSC are independently associated with the prognosis of hepatocellular carcinoma (HCC). Different oncogenes may drive various CSC subtypes identified by different cell surface markers, which challenge the definition of molecular-targeted therapeutic agents (29). It is controversial whether there are bipotent stem cells in breast. ScRNA-Seq data from human mammary epithelial cells revealed a highly efficient cellular state enriched in independent mammary stem cell expression patterns. Bipotent stem-cell-like cells are associated with the clinical outcome of breast cancer, that is, overexpression of regulatory genes $\mathrm{Ybx} 1$ and ENO1 is associated with the risk of breast cancer (39). In addition, assessing the prognostic value of CSC based on single-cell transcriptional data can provide evidence for intratumoral heterogeneity, tumor progression and its clinical significance.

With the application of single-cell technology, researchers have explored and understand transcriptional events in cells of early embryonic development. For example, for the first time, researchers have compared Wilms tumor cells and renal cancer cells with normal renal cells at different developmental stages and ages (fetus, children, adolescents and adults) by scRNA-seq. They found that Wilms tumor cells in pediatric patients share characteristics with specific renal cells in normal development, thus providing evidence for the hypothesis that Wilms tumor cells are abnormal fetal cells. In addition, in adult renal cell carcinoma, researchers have found that renal cancer cells in adult patients originate from a rare healthy adult renal cell PT1 (27). This finding provides a new idea to treat renal cell carcinoma in children by controlling the development of cancer cells rather than killing cancer cells by chemotherapy. These results may lay a foundation for the development of novel treatments for renal cell carcinoma that target PT1 renal cells. Similarly, another study showed that human cerebellar tumor transcriptome is most similar to that in fetal life development. Single-cell transcriptome data highlight cerebellar tumors is a disease of early brain development and provide the most direct evidence for the peak incidence of brain tumors in early childhood (40).

ScRNA-seq was used to construct single-cell maps of cancer cells through analysis data from many samples. For example, scRNA-seq was used to construct a single-cell transcriptome network of cellular and molecular characteristics of gastric epithelial cells with different lesions in a study of EGC (22) 
and to reveal the biological basis of cell development status of each MB subgroup from 25 medulloblastomas (41). These studies have built some single-cell databases and can be used in future studies. With the help of these new system maps, researchers can lock disease-causing genes in special types of cells, develop new treatments for tumors and assess the chance of tumor metastasis and the impact of treatment on the development of cellular state (42).

\section{Study on Genetic Heterogeneity and Phenotypic Variation of Tumor Cells}

Genotype information deduced from scRNA-seq data can help reveal the heterogeneity of genes and their transcriptions as well as their interaction in tumor progression. The researchers used an integrated method for scRNA-seq to analyze the inheritance, expression, and function of 401 specimens from TCGA and obtained a cellular map of the cellular status and genetic diversity of glioblastoma (13). Studies on gene expression heterogeneity with scRNA-seq in MCF7, a common breast cancer cell line, showed that persistent instability was quickly transformed into cell line heterogeneity. In addition, genetic heterogeneity produces unique gene expression patterns, resulting in differential sensitivity of tumor cells to drugs (43).

At the same time, some studies use scRNA-seq to investigate the origin, extent, and outcome of genetic variation, and provide researchers with a framework to understand these variations in order to further study of cancer. Single-cell gene expression data of non-small cell lung cancer showed that when FBXO17 (a negative modulator of glycogenase kinase $3 \beta(44))$ abundance was not under control, it regulated cell proliferation and survival by regulating Akt and ERK kinase activation, thus the potential role of F-box protein in regulating tumorigenesis was proposed (45). Another study performed identified knockdown of RAD51AP1 significantly suppressed tumor volume and prolonged survival in an intracranial EGFRvIII-positive glioma model by analyzing scRNA-seq data from wild-type EGFR cells and mutant EGFRvIII cells (32). ScRNA-seq data also revealed that the known nuclear hormone receptor retinoic-acidreceptor-related orphan receptor gamma $(\mathrm{ROR} \gamma)$ in pancreatic cancer stem cells which drives $\mathrm{T}$ cell differentiation is upregulated during the progression of tumor and its pharmacological inhibitory effect leads to significant defect in the growth and increase in survival rate of pancreatic cancer (14).

Genotypic analysis of gene expression variation by scRNAseq is a useful supplement to the existing methods. Fan J developed a new HoneyBADGER method, which is used together with scRNA-seq to identify loss of heterogeneity and changes in copy number at the single-cell level. By examining data from patients with multiple myeloma (MM), the researchers found that although major gene subclones do show distinct transcriptional features conducive to tumor progression, other prominent aspects independent of the transcriptional heterogeneity of the gene sub-clonal structure may be driven by other mechanisms, including potential variations in epigenetic status or microenvironment (46). Meanwhile, Fasterius E. has also developed a single-cell variant analysis method. This method can be used to compare and cluster cells based on the genetic variation in single nucleotide variants. It also highlights the genetic heterogeneity of the tumor core, heterogeneity between metastases and high levels of variation in driver genes (47). This approach represents a considerable extension of scRNA-seq functionality, allowing researchers to take advantage of all the data generated from their experiments.

\section{Explore the Trajectory of Cell and Gene Development}

The effects of molecular randomness, microenvironment and cell behavior usually lead to significant heterogeneity of cell population, thus blurring the dynamic biological principles that regulate cell state transitions. Single-cell high-throughput technology provides a way to discover these states and their transitions (48). There are many algorithms used in scRNA-seq that can infer the trajectory of a cell. A newly released algorithm, CellRouter (48), is very powerful in modeling the trajectory between the early cell state and the transitional cell state during cell differentiation. As another method of trajectory inference, STREAM can accurately reconstruct complex development trajectories (49). In the absence of surface markers, researchers have also developed genotyping of transcriptome (GoT), which has been used to study how somatic mutations disrupt the complex process of hematopoiesis in humans (50).

\section{Exploring the Developmental Trajectory of Cancer Cells}

Nguyen QH and his colleagues used scRNA-seq and Monocle to analyze the transcriptome of mammary epithelial cells in seven patients undergoing mammoplasty, generated continuous pedigree levels for the Pseudotemporal reconstruction of differentiation trajectory, and linked a basic pedigree closely to two branches of the differentiated lumen pedigree (20). It provided evidence for determining the steady-state differentiation trajectory of adult breast and the origin of different subsets of basic and lumen pedigree. ScRNA-seq identified and characterized tumor plasma cells in low burden disease environment, such as asymptomatic precursor monoclonal gamma globulin disease (MGUS), with high sensitivity and confidence throughout the clinical progression from normal plasma cells in multiple myeloma. And a study suggested using direct molecular detection to track the pathogenesis of early multiple myeloma (51). ScRNA-seq results will provide resources for identifying changes during cancer progression, a basis for promoting cancer prevention strategies, and methods for early cancer detection.

\section{Exploring the Mutation Trajectory of Oncogenes}

Despite detailed information on cancer variants and the frequency of clones, the order of acquisition of these mutations is unknown. The order of mutations in the cells can be elucidated by scRNAseq. For example, CD34 + CD38 - pluripotent progenitor cells and bone marrow cells were analysed by scRNA-seq. It has been revealed that the loss of the fusion gene $\mathrm{CDKN} 2 \mathrm{~A} / \mathrm{B}$ appears in 
the late stages of leukemia, while the mutation of NOTCH1 is a relatively late event (34). Depending on the order in which mutations are acquired, patients can have different clinical manifestations and response to treatment. In addition, scRNA-seq analysis has observed FOXP3 + malignant T cells and GATA3 + or IKZF2 +(HELIOS)tumor cells that are transformed from FOXP3 + T cells during cloning and evolution of Sézary, an aggressive form of cutaneous T-cell lymphoma (52). Similarly, a group of gastric precancerous lesions and early gastric cancer (EGC) specific marker genes were identified by scRNA-seq, for example, OR51E1 is a unique endocrine cell marker in the early stages of malignant lesions, and HES6 may mark the goblet cell cluster, which may be helpful for early identification of metaplasia (22). These genes have clinical value for predicting the early stages of cancer.

\section{PROGRESS IN THE APPLICATION OF SCRNA-SEQ IN TUMOR MICROENVIRONMENT}

Epithelial tissue tumors comprise complex and heterogeneous cell types from different sources, which can be divided into two categories: cancer cells originated from epithelial tissue and stroma cells. Stroma cells can be divided into: Infiltrating immune cells (IIC), cancer-related fibroblasts (CAF), and angiogenic vascular cells (AVC) (Figure 2) (53). Besides these, Jordan A. Ramilowski demonstrated that the normal function of postnatal animals is tightly controlled by intercellular communication and widely depends on the interaction between secreted ligands and cell surface receptors in tumor microenvironment $(54,55)$.

The infiltrating immune cells of tumor are also highly heterogeneous (56). Although cell signal pathway analysis and computational deconvolution analysis may indicate the main infiltrating cells, the results are not detailed enough (57). Methods to study the heterogeneity of tumor stroma cells also include laser dissection/capture and flow cytometry. These methods require prior knowledge of the specific marker genes to be visualized, but the blocking effects of marker genes on endothelial cells (EC), such as DLL4 and VEGF, make it difficult to be labeled (58). However, scRNA-seq results showed the role of VEGF and DLL4-Notch signal transduction can be detected in determining EC phenotype of tumors (46).

Microenvironmental cell types and gene changes are described at unprecedented resolutions by scRNA-seq for a variety of cancers at the single cell level, such as melanoma (59), glioma $(13,21)$, breast cancer $(17)$, head and neck cancer (36), pancreatic cancer (31), and lung cancer (33). Some scientists have used scRNA-seq to construct an immune map of cancer in TME, for example, Elham Azizi constructed an immune map of breast cancer by analyzing immune cells from eight types of breast cancer and normal tissues, blood and lymph nodes (60), and Zheng isolated $\mathrm{T}$ cells from tumors, adjacent normal tissues, and peripheral blood to depict the immune map

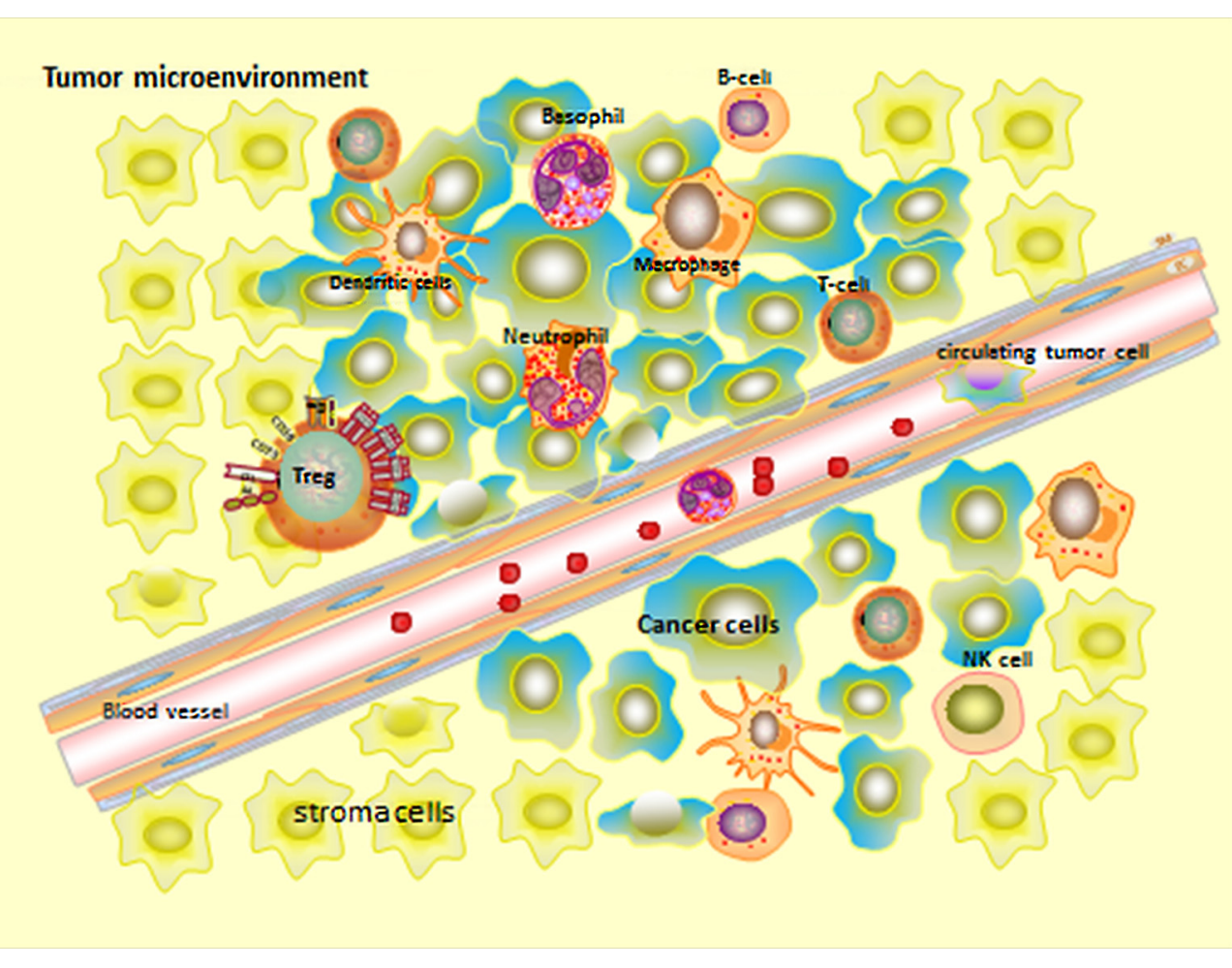

FIGURE 2 | Tumor microenvironment. 
of hepatocellular carcinoma (30). Study also found that Prkarla mutations in tumors result in dramatic changes in the genetic program of cancer cells through scRNA-seq, thus reshaping the microenvironment of the tumor (61).

\section{Tumor-Infiltrating T-lymphocytes}

CD4+ regulatory $\mathrm{T}$ cells (Tregs) expressing transcription factor FOXP3 are highly immunosuppressive, and in malignant tumors, they promote cancer progression by suppressing antitumor immunity (62). Manu P. Kumar identified and compared the ligand-receptor interactions in six homologous mouse tumor models (B16-F10 melanoma, EMT6 breast cancer, LL2 lewis lung cancer, CT26 colon cancer, MC-38 colon cancer and Sa1N fibrosarcomas model) using scRNA-seq data and further quantified ligand-receptor interactions between $\mathrm{T}$ cell subsets and their relationship with immune infiltration (15). ScRNA-seq showed that Tregs and CD8 T cells were more abundant in primary liver tumors than in other tissues, but the majority of these $\mathrm{T}$ cells were in a depleted state, thus revealing why tumor cells escape immune surveillance (30). A class of FOXP3 suppressor $\mathrm{T}$ cells has been found in exhausted CD8 + T cell subsets, suggesting that depleted $\mathrm{T}$ cells may further develop into FOXP3 T cells (30).

ScRNA-seq can find many genes associated with TIL-T. De Simone $\mathrm{M}$ et al. found that several immune checkpoints and their ligand transcripts in tumor-infiltrating Treg cells were upregulated in colorectal cancer or non-small cell lung cancer, and the specific signaling molecules were expressed on cell surface, such as interleukin-1 receptor 2 (IL1R2), programmed death (PD)-1Ligan1, PD-1ligand2, and CCR8 chemokines (63). TIL-T from follicular lymphoma (FL) also co-express genes with immune checkpoint molecules, such as CEBPA and B2M genes (16). Notably, the characteristic genes of Treg cells are highly expressed in whole tumor samples, for example, LAYN, MAGEH1 and CCR8 are associated with poor prognosis (63). $\Gamma \delta \mathrm{T}$ lymphocytes account for about $1 \%$ of human peripheral blood monocytes and have important anticancer functions. ScRNA-seq data can detect human $\gamma \delta$ T lymphocytes from a large complex mixture of cells and specifically detect their $\mathrm{T}$ cell receptor (TCR) subsets, including $T C R v \delta 1$ and $T C R v \delta 2$. In human cancers, $\gamma \delta$ TILs mainly express TCRv $\delta 1$, which is much less in tumor than in blood and has no correlation with the abundance of $\alpha \beta$ TIL (64). These findings provide a basis for an in-depth understanding of the molecular nature and function of human tumor-infiltrating Treg cells.

\section{B Lymphocytes}

The number of B lymphocytes in tumor tissue is large, but the type of B cells in tumor tissue and the presence or absence of subtypes are unknown. A scRNA-seq study of lung cancer showed nine clusters of B cells. Six of these are enriched in tumors: follicular B cells that express high levels of CD20 (MS4A1), CXCR4 and HLA-DR (class 1 and 2), plasma B cells that express immunoglobulin (class 3 and 6), and mucosa-associated lymphoid tissue-derived (MALT) B cells that express immunoglobulin A, M and JCHAIN (class 5 and 7), demonstrating $B$ cell heterogeneity in tumor tissue (65). In another study, the researchers explored a potential transcriptional network of follicular B-cell lymphoma through single-cell transcriptome. Based on gene expression, normal immune subsets and malignant $\mathrm{B}$ cells in each lymphoma were distinguished. Malignant B cells exhibit suppression of immunoglobulin (Ig) light chain (Igא or $\operatorname{Ig} \lambda$ ) expression, upregulation of BCL2, FCER2, CD52 genes, and downregulation of major histocompatibility II genes. Interestingly, a mosaic of malignant B cell subclone coexisting in FL was found (16). ScRNA-seq can infer the B-cell population that other analytical methods cannot detect.

\section{Cancer-Related Fibroblasts}

In some studies, the heterogeneity of cancer-related fibroblasts (CAFs) of various types of cancers has been demonstrated by scRNA-seq, and different subsets affect tumor microenvironment. For instance, Baryawno $\mathrm{N}$ demonstrated that one of the five fibroblast subpopulations in bone marrow expressed Cxcl12, and the fibroblasts expressing Cxcl12 were associated with invasive solid tumors (10). In colorectal cancer analyzed by scRNA-seq, there are two different CAF subtypes, CAF-A and CAF-B. Experimental results show that only CAF-A cells express fibroblast activation protein (FAP), a membrane-serine protease expressed only in CAF, so targeted treatment of FAP can be carried out based on the heterogeneity of CAF (65). Seurat divided CAFs from COLO205 tumor into six clusters and three different subpopulations, which were fibroblast-like cells, smooth muscle-like cells, and peripheral cells, respectively. It was found that two genes, Notch3 and Angpt2, which may be involved in angiogenesis, were highly expressed in peripheral cells. CAF can promote tumor angiogenesis by secreting feedback molecules during local hypoxia, and may be resistaant to angiogenic drugs (58).

In addition to immune cells themselves, CAF also plays a crucial role in immune escape and cancer metastasis. Studies have shown that upregulation of epithelial-mesenchymal transition (EMT)-related genes is driven by changes in expression in fibroblasts in colorectal tumors (65). In head and neck cancer, tumors with high CAF scores and high p-EMT scores have a particularly high tendency to metastasize, which may reflect that paracrine signaling between CAF and malignant cells can promote lymph node metastasis (36). In addition, it was found that a group of genes specifically expressed in CAF and closely associated with $\mathrm{T}$ cell infiltration included a variety of complementary factors ( $\mathrm{cls}, \mathrm{clr}, \mathrm{c} 3 \mathrm{a}, \mathrm{cfb}$ and $\mathrm{clnh}$ ) in a study of melanoma. These results suggest that complement activity may be related to the regulation and recruitment of $\mathrm{T}$ cell-mediated anti-tumor immune responses, which provides clues for further study of the cellular and molecular mechanisms by which CAF recruits $\mathrm{T}$ cells (59).

\section{Tumor Infiltrating Myeloid Cells}

TIMs contain monocytes, macrophages, dendritic cells, and neutrophils, which can diversify into a variety of states that can promote or limit tumor growth and become key regulators. ScRNA-seq was used to localize TIMs in patients with non-small cell lung cancer (NSCLC) and to analyze TIM in mice. Twentyfive TIM states were found, most of which could be found in different patient samples (66). Moreover, changes in myeloid cell population in tumors originated from the functional branch 
point of the infiltrating circulation affect monocytes, rather than the reprogramming of macrophages in mature tumor (67).

Zhu YP and colleagues used a scRNA-seq approach to show that mononuclear neutrophil progenitor cells (hNeP) significantly increase tumor growth after metastasis. In addition, hNeP is found in the blood of patients who have recently been diagnosed with melanoma, suggesting that $\mathrm{hNeP}$ can be used as a biomarker for early cancer detection, especially for cancers in which neutrophil levels and function are important (68). Tumor-associated macrophages (TAM) originate from myeloid precursors (69). TAMs, commonly known as polarized M2 macrophage populations, are cells with immunosuppressive and tumorigenic functions (70) and express arginase 1 (Arg1). It was found that there are two morphological subsets, Arg1+ TAM and Arg1- TAM, and that the pharmacological inhibitory effect of Arg1 is not synergistic with anti-programmed cell death 1 (aPD-1) therapy (71). Phenotypic differences in TAM of different lineages are distinguished by the scRNA-seq of human glioma. Hematogenous TAM is abundant in peripheral blood vessels and necrotic areas before treatment and significantly infiltrates gliomas, and its gene markers are related to the survival rate of gliomas and its infiltration varies with the subtypes of glioma. TAM preferentially expresses immunosuppressive cytokines (21). Moreover, autocrine interactions between glioma cells were identified. In addition, IL-8 is mainly expressed by TAM, and its receptor SDC1 is highly expressed in glioma stem cell-like cells (55).The results suggest that immunotherapy with immunosuppressive blood-derived TAM should be selected as a target.

\section{RESEARCH ON CIRCULATING TUMOR CELL}

Circulating tumor cell (CTC) not only provides an important mechanism for cancer metastasis, but also offers the possibility of diagnosing and monitoring cancer simply (72). In addition, repeated and quantitative analysis of nucleic acids in CTC can help to understand changes in clonal composition over time, enabling dynamic treatment (73). Studies have demonstrated the advantages of single-cell transcriptional profiling in detecting CTCs provide a new tool for the development of cancer biomarkers using liquid biopsy technology.

Because it is not feasible to evaluate IGF2 disorders (a kind of carcinogenic driver) by detecting tumor mutations alone, scRNA-seq of CTC is an excellent tool for detecting non-mutable drug-related gene abnormalities, such as IGF2 overexpression (28). CTC gene expression profiles of patients with Alveolar Rhabdomyosarcoma (ARMS) were analyzed by scRNA-seq. Of the top 150 genes with the greatest difference, 70 genes were expressed at a significant level in the CTC population. These genes have previously been shown to be associated with metastasis, but have never been detected in ARMS (35). This may suggest novel genes for ARMS diagnosis.

ScRNA-seq of breast cancer CTCs identified them into two types: one with estrogen responsiveness and another with EMT characteristic (74). Another study used a scalable hydrodynamic scRNA-seq bar coding technique called Hydro-Seq to resolve the contamination of blood cells. They identified cells that express markers of epithelial/mesenchymal cell state transitions and detected drug targets of breast cancer CTCs (75). In the future, scRNA-seq is expected to help physicians select appropriate anticancer drugs and treatments, and monitor the progression of the disease and the therapeutic effect at any time, which will help personalized treatment for patients.

\section{RESEARCH IN CANCER THERAPY}

Chemotherapy, targeted therapy and immunotherapy have always been vital weapons in the fight against cancer. Due to the importance of ligand-receptor interactions for patient prognosis, such as ipilimumab, an immunosuppressive agent targeting CD28 or CTLA4, and pembrolizumab and nivolumab targeting PD1 or PDL1, therapeutic agents targeting immunization checkpoints have become a promising approach in clinical treatment (76). High-dimensional analysis can not only help to identify the best combination of a variety of available immunotherapy drugs, but also identify potential new reactive biomarkers, so that cancer immunotherapy is more effective, specific, and safe than the previous one available to us.

Cancer cells will develop resistance and cross-resistance to a variety of chemotherapeutic drugs that have unrelated functions and structures after exposure to chemotherapeutic drugs (77). In fully active and symptomatic diseases, the frequent identification of the precise molecular characteristics and polyclonal structure of malignant state by scRNA-seq provides a better understanding of the mechanisms of resistance after treatment (51). ScRNA-seq studies have also revealed a high-resolution picture of drug-resistant cells to immune checkpoint inhibitors (ICIs), which provides a framework for studying cell-cell interactions and drug interactions in other tumor ecosystems.

\section{Targeted Therapy}

Resistance to targeted BRAF inhibitors is widely existed in melanoma (78). Using scRNA-seq analysis and cluster assessment (SAKE) to track melanoma cells that have developed resistance to BRAF inhibitors, several BRAF inhibitor resistance markers have been obtained, and new resistance markers have been identified in very few cell populations before using drug (24). Some scRNA-seq data confirm that neural c stem cells (NCSC) are the major driving force for drug resistance. Application of ScRNA-seq to patientderived melanoma minimal residual disease (MRD) of BRAF mutant xenotransplantation identified up to four different drugresistant transcriptional states. One of them showed the NCSC transcriptional program driven mainly by the nuclear receptor RXRG. RXR antagonists reduced the accumulation of NCSC in $\mathrm{MRD}$ and delayed the development of resistance (23).

ScRNA-seq reveals the rationale for improving biomarkers and providing new treatments to patients, and suggests new therapeutic strategies to overcome drug resistance in immunotherapy.Two patients with metastatic Merkel cell carcinoma (MCC) treated 
with T-cell immunotherapy and immune checkpoint inhibitors (ICIs) were also studied with scRNA-seq. It was observed that the injected CD8 T cells infiltrated into the reduced MCC, and the tumor regression was mediated by supporting $\mathrm{T}$ cells. By targeting the selective transcription loss of human leukocyte antigen (HLA) under T-cell pressure to limit targeting the epitopes of Merkel cell polyomavirus $(\mathrm{MCPyV})$, the difference from the genetic loss of HLA is that drug treatment may reverse the inhibitory action of tumor-specific HLA (25). Transcriptional inhibition of class I loci may be the basis of resistance to other immunotherapy (including checkpoint inhibitors). In addition, another study applied scRNAseq and identified a malignant cellular program related to $\mathrm{T}$ cell rejection and ICI resistance prediction in melanoma. It demonstrated that CDK4/6 inhibitors can inhibit this program and make melanoma tumor in a mouse model sensitive to ICI (79).

\section{Chemotherapy}

Therapies that target the epidermal growth factor receptor (EGFR) have variable and unpredictable effects in breast cancer. Cell subsets with EGFR inhibitory response to gefitinib were identified by scRNA-seq and EGFR ${ }^{\text {hi }}$ subsets showed enhanced stem cell-like characteristics in three negative breast cancer (TNBC) (18). Heterogeneous expression of EGFR is associated with the sensitivity to gefitinib, which provides a basis for further treatment planning.

In a TNBC mouse model, scRNA-seq data revealed that the hedgehog $(\mathrm{Hh})$ signal from the fibroblast (CAF) binds to $\mathrm{Hh}$ ligands from tumor cells, which promotes FGF5 expression and collagen remodeling in the matrix, resulting in the production of cancer stem cells (CSCs) with chemoresistant phenotype. Matrix therapy with smoothened inhibitors (SMOi) for patient-derived xenografts can down-regulate the expression of CSC markers and make the tumor sensitive to Docetaxel, thereby significantly increasing the survival rate and reducing the rate of metastasis (19). At the same time, the hedgehog signal sent by CAF will be a new plastic mediator for CSC and a new therapeutic target for TNBC.

\section{Immunotherapy}

In the present study, scRNA-seq addresses the challenges of insufficient understanding of the complexity of immune cell subtypes and possible differences in the immune system between species, which lays the foundation for studying the potential of immune cell as a target of immunotherapy in the future. ScRNA-seq shows that the effects of different immune checkpoint therapy (ICT) on monocytes/macrophages in tumors are particularly significant, and they are partially dependent on IFN $\gamma$ and change over time, which leads to a high degree of plasticity and complexity of the cell population (67). These insights into the transcriptional, molecular, and functional changes that occur within the immune cells of major tumors after cancer immunotherapy strongly support the need to simultaneously consider both innate (e.g., macrophage) immunity and adaptive (e.g., CD4 and CD8 T cells) immunity to improve the efficacy of cancer immunotherapy.

ScRNA-seq can explore the effects of certain cytokines on immune cell development and discover some important novel immune cells that have not been revealed (80). For example,
scRNA-seq and lineage tracing identified a population of TCF- 1 + Ly108+ PD-1+ CD8 T cells and revealed that TCF-1 mediated Tbet-to-Eomes transcription factor conversion in the culture of exhausted CD8 T cell (Tex) precursors, and PD-1 was identified as a protector of early TCF-1 subgroup (81). Congenital lymphoid cells (ILCs) are a newly identified family of innate immune cells. ScRNA-seq revealed that differentially expressed ikzf3 in human ILC1 is a known target of immunomodulatory drug (IMiD) -mediated degradation such as lenalidomide or pomalidomide, which increases the possibility that ILC may also be a IMiD mediated immunomodulatory target (82). Kubli SP et al. used single-cell transcriptome analysis to confirm that FCMR (a putative receptor for soluble IgM) that plays an important role in regulating immune responses during autoimmunity generally acts to limit DC maturation in the TME and then suppresses antitumor T cell responses (83). Blockage of FCMR and synergistic treatment with T cell-specific anti-PD1 in myeloid cells can inhibit the growth of B16 melanoma (83).

The blocking of the reactivation of immune response by PD1, a marker of T-cell exhaustion, is becoming a promising cancer treatment. The recurrence of hematological malignancies after allogeneic stem cell transplantation (allo-SCT) limits the success of this approach. Because PD-1 expression may differ from that of non-transplant individuals, its blocking may lead to graftversus-host disease (GVHD). The kinetics of T cell exhaustion and its relationship with leukemia recurrence were analyzed by scRNA-seq in patients undergoing allo-SCT. Although leukemia antigen-specific $\mathrm{T}$ cells do not overexpress PD-1, LAG3 and TIM3 are over expressed during relapse (26). So we could target LAG3 and TIM3 as a new therapy approach.

\section{CONCLUSION}

Despite the many advantages of scRNA-seq, certain limitations and challenges of scRNA-seq cannot be avoided. In single-cell level studies, the main problem is that the starting amount of RNA is low and the reverse transcriptome is not easily amplified (46). Another major problem with scRNA-seq is the increase in impurity levels in the abundance of transcripts measured. At the same time, excessive transcript loss rates and random transcription events will result in a large amount of data, high variability, and complex expression distribution undetected. Therefore, it is important to distinguish low quality, high impurity samples with poor amplification or degradation in the process of library preparation (24). Data on the use of single-cell techniques to analyze the genome-wide transcriptome of CTCs are scarce. Due to the rarity of CTC in blood (about 0-10 CTCs in 7,000,000 nucleated cells), we need to enrich CTC in large numbers before single-cell sequencing. ScRNAseq strongly implies the origin of tumors in isolated cells, but the low coverage and dispersion of these data make it impossible to clearly assess malignant characteristics, such as somatic mutations or chromosomal aberrations (28).

Single-cell-range technologies offer the advantage of measuring multiple molecules such as DNA, RNA, proteins, and chromatin, at high resolution. By isolating different types of molecules from a single cell simultaneously, they can be analyzed 
in parallel (11). For example, the combination of whole genome and whole transcriptome sequencing (scGT-seq) can help establish the relationship between genomic changes in cancer and their effects on immune cells (84), such as studies on gastric cancer and primary gastric tumors (85). A combination of singlecell DNA methylation and transcriptome sequencing (scMTseq) found that Low-methylated regions (LMR) showed significant difference in methylation levels, which is consistent with their role as remote regulatory elements to control gene expression (84). ScTrio-Seq has further developed single-cell multi-omics technologies by combining three omics methods, genomics, transcriptomics, and epigenomics. The spatial information of individual cells in a tissue is usually lost during the isolation step, so individual cell sequencing data usually do not show how cells organize to achieve coordinated functions within the target tissue (26). The field of single-cell spatial transcription is under intensive investigation, and many new technologies have been developed to maintain or restore spatial information of sequenced single cells, such as seqFISH (sequential fluorescence in situ hybridization of RNA) (86), MERFISH (Multiplexed error-robust fluorescence in situ hybridization) (87), FISSEQ (fluorescent in situ sequencing) (88), or TIVA (Transcriptome in vivo analysis) (89). All of these methods can identify interactions between different cell types by examining genes expressed in vivo in the context of specific tissue structures.

ScRNA-seq technology can produce a lot of data, some current bioinformatics tools need to be used to analyze these data (90). Principal component analysis (PCA) was performed to reduce the dimensionality on the log transformed gene-barcode matrices of top variable genes. Cells were clustered based on a graph-based clustering approach and were visualized in 2-dimension using t-SNE. R package SingleR, a novel computational method for

\section{REFERENCES}

1. Zhang X, Marjani SL, Hu Z, Weissman SM, Pan X, Wu S. Single-Cell Sequencing for Precise Cancer Research: Progress and Prospects. Cancer Res (2016) 76:1305-12. doi: 10.1158/0008-5472.CAN-15-1907

2. Tang F, Barbacioru C, Wang Y, Nordman E, Lee C, Xu N, et al. mRNA-Seq whole-transcriptome analysis of a single cell. Nat Methods (2009) 6:377-82. doi: $10.1038 /$ nmeth. 1315

3. Klein AM, Mazutis L, Akartuna I, Tallapragada N, Veres A, Li V, et al. Droplet barcoding for single-cell transcriptomics applied to embryonic stem cells. Cell (2015) 161:1187-201. doi: 10.1016/j.cell.2015.04.044

4. Macosko EZ, Basu A, Satija R, Nemesh J, Shekhar K, Goldman M, et al. Highly Parallel Genome-wide Expression Profiling of Individual Cells Using Nanoliter Droplets. Cell (2015) 161:1202-14. doi: 10.1016/j.cell.2015.05.002

5. Zheng GXY, Terry JM, Belgrader P, Ryvkin P, Bent ZW, Wilson R, et al. Massively parallel digital transcriptional profiling of single cells. Nat Commun (2017) 8:14049. doi: 10.1038/ncomms14049

6. Valihrach L, Androvic P, Kubista M. Platforms for Single-Cell Collection and Analysis. Int J Mol Sci (2018) 19:807. doi: 10.3390/ijms19030807

7. Zilionis R, Nainys J, Veres A, Savova V, Zemmour D, Klein AM, et al. Singlecell barcoding and sequencing using droplet microfluidics. Nat Protoc (2017) 12:44-73. doi: 10.1038/nprot.2016.154

8. Haque A, Engel J, Teichmann SA, Lönnberg T. A practical guide to single-cell RNA-sequencing for biomedical research and clinical applications. Genome Med (2017) 9(1):75. doi: 10.1186/s13073-017-0467-4 unbiased cell type recognition of scRNA-seq, is used to infer the cell of origin of each of the single cells independently and identify cell types (91). Using Monocle algorithm can measure a cell's biological progression, which is called "pseudotime" (92). Further technical improvements in bioinformatics tools will greatly facilitate the applications of scRNA-seq.

The continuous development of single-cell transcriptome technology and its combination with multi-domain technologies and algorithms will bring new revolutions to next generation genome sequencing. At the same time, its unique role in the field of cancer will help us explain biological mechanisms that could not be elucidated before and greatly promote the development of precision medicine, bringing new breakthroughs in clinical diagnosis, treatment, and prognosis of patients.

\section{AUTHOR CONTRIBUTIONS}

JL wrote the manuscript and collected the literature. TX collected the literature and modified the manuscript. YJ collected the literature. $\mathrm{BH}$ proposed the amendments. YZ designed the content of the article and proposed amendments. All authors contributed to the article and approved the submitted version.

\section{FUNDING}

The work was supported by the National Natural Science Foundation of China (81772772) and the Department of Science and Technology, Jilin Province, China (CN) (20190905004SF, 20170622008JC).

9. Wu Y, Zhang K. Tools for the analysis of high-dimensional single-cell RNA sequencing data. Nat Rev Nephrol (2020) 16:408-21. doi: 10.1038/s41581020-0262-0

10. Baryawno N, Przybylski D, Kowalczyk MS, Kfoury Y, Severe N, Gustafsson K, et al. A Cellular Taxonomy of the Bone Marrow Stroma in Homeostasis and Leukemia. Cell (2019) 177:1915-1932 e1916. doi: 10.1016/j.cell.2019.04.040

11. Ren X, Kang B, Zhang Z. Understanding tumor ecosystems by single-cell sequencing: promises and limitations. Genome Biol (2018) 19:211. doi: 10.1186/s13059-018-1593-z

12. Heymann D, Tellez-Gabriel M. Circulating Tumor Cells: The Importance of Single Cell Analysis. Adv Exp Med Biol (2018) 1068:45-58. doi: 10.1007/978-981-13-0502-3_5

13. Neftel C, Laffy J, Filbin MG, Hara T, Shore ME, Rahme GJ, et al. An Integrative Model of Cellular States, Plasticity, and Genetics for Glioblastoma. Cell (2019) 178:835-49 e821. doi: 10.1016/j.cell.2019.06.024

14. Lytle NK, Ferguson LP, Rajbhandari N, Gilroy K, Fox RG, Deshpande A, et al. A Multiscale Map of the Stem Cell State in Pancreatic Adenocarcinoma. Cell (2019) 177:572-586 e522. doi: 10.1016/j.cell.2019.03.010

15. Kumar MP, Du J, Lagoudas G, Jiao Y, Sawyer A, Drummond DC, et al. Analysis of Single-Cell RNA-Seq Identifies Cell-Cell Communication Associated with Tumor Characteristics. Cell Rep (2018) 25:1458-1468 e1454. doi: 10.1016/j.celrep.2018.10.047

16. Andor N, Simonds EF, Czerwinski DK, Chen J, Grimes SM, Wood-Bouwens C, et al. Single-cell RNA-Seq of follicular lymphoma reveals malignant B-cell types and coexpression of T-cell immune checkpoints. Blood (2019) 133:1119-29. doi: 10.1182/blood-2018-08-862292 
17. Savas P, Virassamy B, Ye C, Salim A, Mintoff CP, Caramia F, et al. Single-cell profiling of breast cancer $\mathrm{T}$ cells reveals a tissue-resident memory subset associated with improved prognosis. Nat Med (2018) 24:986-93. doi: 10.1038/ s41591-018-0078-7

18. Savage P, Blanchet-Cohen A, Revil T, Badescu D, Saleh SMI, Wang YC, et al. A Targetable EGFR-Dependent Tumor-Initiating Program in Breast Cancer. Cell Rep (2017) 21:1140-9. doi: 10.1016/j.celrep.2017.10.015

19. Cazet AS, Hui MN, Elsworth BL, Wu SZ, Roden D, Chan CL, et al. Targeting stromal remodeling and cancer stem cell plasticity overcomes chemoresistance in triple negative breast cancer. Nat Commun (2018) 9:2897. doi: 10.1530/ oncolabs.1.P009

20. Nguyen QH, Pervolarakis N, Blake K, Ma D, Davis RT, James N, et al. Profiling human breast epithelial cells using single cell RNA sequencing identifies cell diversity. Nat Commun (2018) 9:2028. doi: 10.1038/s41467-018-04334-1

21. Muller S, Kohanbash G, Liu SJ, Alvarado B, Carrera D, Bhaduri A, et al. Single-cell profiling of human gliomas reveals macrophage ontogeny as a basis for regional differences in macrophage activation in the tumor microenvironment. Genome Biol (2017) 18:234. doi: 10.1186/s13059-017-1362-4

22. Zhang P, Yang M, Zhang Y, Xiao S, Lai X, Tan A, et al. Dissecting the SingleCell Transcriptome Network Underlying Gastric Premalignant Lesions and Early Gastric Cancer. Cell Rep (2019) 27:1934-1947 e1935. doi: 10.1016/ j.celrep.2019.04.052

23. Rambow F, Rogiers A, Marin-Bejar O, Aibar S, Femel J, Dewaele M, et al. Toward Minimal Residual Disease-Directed Therapy in Melanoma. Cell (2018) 174:843-855 e819. doi: 10.1016/j.cell.2018.06.025

24. Ho YJ, Anaparthy N, Molik D, Mathew G, Aicher T, Patel A, et al. Single-cell RNA-seq analysis identifies markers of resistance to targeted BRAF inhibitors in melanoma cell populations. Genome Res (2018) 28:1353-63. doi: 10.1101/ gr.234062.117

25. Paulson KG, Voillet V, McAfee MS, Hunter DS, Wagener FD, Perdicchio M, et al. Acquired cancer resistance to combination immunotherapy from transcriptional loss of class I HLA. Nat Commun (2018) 9:3868. doi: 10.1038/s41467-018-06300-3

26. Jain P, Tian X, Cordes S, Chen J, Cantilena CR, Bradley C, et al. Overexpression of PD-1 Does Not Predict Leukemic Relapse after Allogeneic Stem Cell Transplantation. Biol Blood Marrow Transplant (2019) 25:216-22. doi: 10.1016/j.bbmt.2018.09.037

27. Young MD, Mitchell TJ, Vieira Braga FA, Tran MGB, Stewart BJ, Ferdinand JR, et al. Single-cell transcriptomes from human kidneys reveal the cellular identity of renal tumors. Science (2018) 361:594-9. doi: 10.1126/science.aat1699

28. D'Avola D, Villacorta-Martin C, Martins-Filho SN, Craig A, Labgaa I, von Felden J, et al. High-density single cell mRNA sequencing to characterize circulating tumor cells in hepatocellular carcinoma. Sci Rep (2018) 8:11570. doi: 10.1038/s41598-018-30047-y

29. Zheng H, Pomyen Y, Hernandez MO, Li C, Livak F, Tang W, et al. Single-cell analysis reveals cancer stem cell heterogeneity in hepatocellular carcinoma. Hepatology (2018) 68:127-40. doi: 10.1002/hep.29778

30. Zheng C, Zheng L, Yoo JK, Guo H, Zhang Y, Guo X, et al. Landscape of Infiltrating $\mathrm{T}$ Cells in Liver Cancer Revealed by Single-Cell Sequencing. Cell (2017) 169:1342-56 e1316. doi: 10.1016/j.cell.2017.05.035

31. Peng J, Sun BF, Chen CY, Zhou JY, Chen YS, Chen H, et al. Single-cell RNAseq highlights intra-tumoral heterogeneity and malignant progression in pancreatic ductal adenocarcinoma. Cell Res (2019) 29:725-38. doi: 10.1038/ s41422-019-0195-y

32. Wang QX, Tan YL, Fang C, Zhou JH, Wang YF, Zhao K, et al. Single-cell RNA-seq reveals RAD51AP1 as a potent mediator of EGFRvIII in human glioblastomas. Aging-Us (2019) 11:7707-22. doi: 10.18632/aging.102282

33. Lambrechts D, Wauters E, Boeckx B, Aibar S, Nittner D, Burton O, et al. Phenotype molding of stromal cells in the lung tumor microenvironment. Nat Med (2018) 24:1277-89. doi: 10.1038/s41591-018-0096-5

34. De Bie J, Demeyer S, Alberti-Servera L, Geerdens E, Segers H, Broux M, et al. Single-cell sequencing reveals the origin and the order of mutation acquisition in T-cell acute lymphoblastic leukemia. Leukemia (2018) 32:1358-69. doi: 10.1038/s41375-018-0127-8

35. Hayashi M, Zhu P, McCarty G, Meyer CF, Pratilas CA, Levin A, et al. Sizebased detection of sarcoma circulating tumor cells and cell clusters. Oncotarget (2017) 8:78965-77. doi: 10.18632/oncotarget.20697
36. Puram SV, Tirosh I, Parikh AS, Patel AP, Yizhak K, Gillespie S, et al. Single-Cell Transcriptomic Analysis of Primary and Metastatic Tumor Ecosystems in Head and Neck Cancer. Cell (2017) 171:1611-24 e1624. doi: 10.1016/j.cell.2017.10.044

37. McGranahan N, Swanton C. Biological and therapeutic impact of intratumor heterogeneity in cancer evolution. Cancer Cell (2015) 27:15-26. doi: 10.1016/ j.ccell.2014.12.001

38. Kim N, Chung W, Eum HH, Lee HO, Park WY. Alternative polyadenylation of single cells delineates cell types and serves as a prognostic marker in early stage breast cancer. PloS One (2019) 14:e0217196. doi: 10.1371/journal.pone.0217196

39. Chen W, Morabito SJ, Kessenbrock K, Enver T, Meyer KB, Teschendorff AE. Single-cell landscape in mammary epithelium reveals bipotent-like cells associated with breast cancer risk and outcome. Commun Biol (2019) 2:306. doi: 10.1038/s42003-019-0554-8

40. Vladoiu MC, El-Hamamy I, Donovan LK, Farooq H, Holgado BL, Sundaravadanam Y, et al. Childhood cerebellar tumours mirror conserved fetal transcriptional programs. Nature (2019) 572:67-73. doi: 10.1038/ncomms8866

41. Hovestadt V, Smith KS, Bihannic L, Filbin MG, Shaw ML, Baumgartner A, et al. Resolving medulloblastoma cellular architecture by single-cell genomics. Nature (2019) 572:74-9. doi: 10.1186/s12964-019-0363-1

42. Zeisel A, Hochgerner H, Lonnerberg P, Johnsson A, Memic F, van der Zwan J, et al. Molecular Architecture of the Mouse Nervous System. Cell (2018) 174:999-1014 e1022. doi: 10.1016/j.cell.2018.06.021

43. Ben-David U, Siranosian B, Ha G, Tang H, Oren Y, Hinohara K, et al. Genetic and transcriptional evolution alters cancer cell line drug response. Nature (2018) 560:325-30. doi: 10.1038/s41586-018-0409-3

44. Suber T, Wei J, Jacko AM, Nikolli I, Zhao Y, Zhao J, et al. SCF(FBXO17) E3 ligase modulates inflammation by regulating proteasomal degradation of glycogen synthase kinase-3beta in lung epithelia. J Biol Chem (2017) 292:7452-61. doi: 10.1074/jbc.M116.771667

45. Suber TL, Nikolli I, O'Brien ME, Londino J, Zhao J, Chen K, et al. FBXO17 promotes cell proliferation through activation of Akt in lung adenocarcinoma cells. Respir Res (2018) 19:206. doi: 10.1186/s12931-018-0910-0

46. Fan J, Lee HO, Lee S, Ryu DE, Lee S, Xue C, et al. Linking transcriptional and genetic tumor heterogeneity through allele analysis of single-cell RNA-seq data. Genome Res (2018) 28:1217-27. doi: 10.1101/gr.228080.117

47. Fasterius E, Uhlen M, Al-Khalili Szigyarto C. Single-cell RNA-seq variant analysis for exploration of genetic heterogeneity in cancer. Sci Rep (2019) 9:9524. doi: 10.1038/s41598-019-45934-1

48. Lummertz da Rocha E, Rowe RG, Lundin V, Malleshaiah M, Jha DK, Rambo $\mathrm{CR}$, et al. Reconstruction of complex single-cell trajectories using CellRouter. Nat Commun (2018) 9:892. doi: 10.1038/s41467-018-03214-y

49. Chen H, Albergante L, Hsu JY, Lareau CA, Lo Bosco G, Guan J, et al. Singlecell trajectories reconstruction, exploration and mapping of omics data with STREAM. Nat Commun (2019) 10:1903. doi: 10.1038/s41467-019-09670-4

50. Nam AS, Kim KT, Chaligne R, Izzo F, Ang C, Taylor J, et al. Somatic mutations and cell identity linked by Genotyping of Transcriptomes. Nature (2019) 571:355-60. doi: 10.1038/s41586-019-1367-0

51. Ledergor G, Weiner A, Zada M, Wang SY, Cohen YC, Gatt ME, et al. Single cell dissection of plasma cell heterogeneity in symptomatic and asymptomatic myeloma. Nat Med (2018) 24:1867-76. doi: 10.1038/s41591-018-0269-2

52. Borcherding N, Voigt AP, Liu V, Link BK, Zhang W, Jabbari A. Single-Cell Profiling of Cutaneous T-Cell Lymphoma Reveals Underlying Heterogeneity Associated with Disease Progression. Clin Cancer Res (2019) 25:2996-3005. doi: 10.1158/1078-0432.CCR-18-3309

53. Hanahan D, Coussens LM. Accessories to the crime: functions of cells recruited to the tumor microenvironment. Cancer Cell (2012) 21:309-22. doi: 10.1016/j.ccr.2012.02.022

54. Ramilowski JA, Goldberg T, Harshbarger J, Kloppmann E, Lizio M, Satagopam VP, et al. A draft network of ligand-receptor-mediated multicellular signalling in human. Nat Commun (2015) 6:7866. doi: $10.1038 /$ ncomms8866

55. Yuan D, Tao Y, Chen G, Shi T. Systematic expression analysis of ligandreceptor pairs reveals important cell-to-cell interactions inside glioma. Cell Commun Signal (2019) 17:48. doi: 10.1186/s12964-019-0363-1

56. Burkholder B, Huang RY, Burgess R, Luo S, Jones VS, Zhang W, et al. Tumorinduced perturbations of cytokines and immune cell networks. Biochim Biophys Acta (2014) 1845:182-201. doi: 10.1016/j.bbcan.2014.01.004 
57. Racle J, de Jonge K, Baumgaertner P, Speiser DE, Gfeller D. Simultaneous enumeration of cancer and immune cell types from bulk tumor gene expression data. Elife (2017) 6:e26476. doi: 10.7554/eLife.26476

58. Zhao Q, Eichten A, Parveen A, Adler C, Huang Y, Wang W, et al. Single-Cell Transcriptome Analyses Reveal Endothelial Cell Heterogeneity in Tumors and Changes following Antiangiogenic Treatment. Cancer Res (2018) 78:2370-82. doi: 10.1158/0008-5472.CAN-17-2728

59. Tirosh I, Izar B, Prakadan SM, Wadsworth MH,2, Treacy D, Trombetta JJ, et al. Dissecting the multicellular ecosystem of metastatic melanoma by singlecell RNA-seq. Science (2016) 352:189-96. doi: 10.1016/j.cell.2018.09.030

60. Azizi E, Carr AJ, Plitas G, Cornish AE, Konopacki C, Prabhakaran S, et al. SingleCell Map of Diverse Immune Phenotypes in the Breast Tumor Microenvironment. Cell (2018) 174:1293-1308 e1236. doi: 10.1016/j.cell.2018.05.060

61. Codina A, Renauer PA, Wang G, Chow RD, Park JJ, Ye H, et al. Convergent Identification and Interrogation of Tumor-Intrinsic Factors that Modulate Cancer Immunity In Vivo. Cell Syst (2019) 8:136-51 e137. doi: 10.1016/ j.cels.2019.01.004

62. Takeuchi $Y$, Nishikawa $H$. Roles of regulatory $T$ cells in cancer immunity. Int Immunol (2016) 28:401-9. doi: 10.1093/intimm/dxw025

63. De Simone M, Arrigoni A, Rossetti G, Gruarin P, Ranzani V, Politano C, et al. Transcriptional Landscape of Human Tissue Lymphocytes Unveils Uniqueness of Tumor-Infiltrating T Regulatory Cells. Immunity (2016) 45:1135-47. doi: 10.1016/j.immuni.2016.10.021

64. Pizzolato G, Kaminski H, Tosolini M, Franchini DM, Pont F, Martins F, et al. Single-cell RNA sequencing unveils the shared and the distinct cytotoxic hallmarks of human TCRVdelta1 and TCRVdelta2 gammadelta T lymphocytes. Proc Natl Acad Sci U S A (2019) 116:11906-15. doi: 10.1073/ pnas. 1818488116

65. Li H, Courtois ET, Sengupta D, Tan Y, Chen KH, Goh JJL, et al. Reference component analysis of single-cell transcriptomes elucidates cellular heterogeneity in human colorectal tumors. Nat Genet (2017) 49:708-18. doi: $10.1038 /$ ng.3818

66. Zilionis R, Engblom C, Pfirschke C, Savova V, Zemmour D, Saatcioglu HD, et al. Single-Cell Transcriptomics of Human and Mouse Lung Cancers Reveals Conserved Myeloid Populations across Individuals and Species. Immunity (2019) 50:1317-1334 e1310. doi: 10.1016/j.immuni.2019.03.009

67. Gubin MM, Esaulova E, Ward JP, Malkova ON, Runci D, Wong P, et al. HighDimensional Analysis Delineates Myeloid and Lymphoid Compartment Remodeling during Successful Immune-Checkpoint Cancer Therapy. Cell (2018) 175:1014-1030 e1019. doi: 10.1016/j.cell.2018.09.030

68. Zhu YP, Padgett L, Dinh HQ, Marcovecchio P, Blatchley A, Wu R, et al. Identification of an Early Unipotent Neutrophil Progenitor with Pro-tumoral Activity in Mouse and Human Bone Marrow. Cell Rep (2018) 24:2329-41 e2328. doi: 10.1016/j.celrep.2018.07.097

69. Ngambenjawong C, Gustafson HH, Pun SH. Progress in tumor-associated macrophage (TAM)-targeted therapeutics. Adv Drug Delivery Rev (2017) 114:206-21. doi: 10.1016/j.addr.2017.04.010

70. Mantovani A, Sozzani S, Locati M, Allavena P, Sica A. Macrophage polarization: tumor-associated macrophages as a paradigm for polarized M2 mononuclear phagocytes. Trends Immunol (2002) 23:549-55. doi: 10.1016/S1471-4906(02)02302-5

71. Arlauckas SP, Garren SB, Garris CS, Kohler RH, Oh J, Pittet MJ, et al. Arg1 expression defines immunosuppressive subsets of tumor-associated macrophages. Theranostics (2018) 8:5842-54. doi: 10.7150/thno.26888

72. Huang X, Liu S, Wu L, Jiang M, Hou Y. High Throughput Single Cell RNA Sequencing, Bioinformatics Analysis and Applications. Adv Exp Med Biol (2018) 1068:33-43. doi: 10.1007/978-981-13-0502-3_4

73. von Bubnoff N. Liquid Biopsy: Approaches to Dynamic Genotyping in Cancer. Oncol Res Treat (2017) 40:409-16. doi: 10.1159/000478864

74. Brechbuhl HM, Vinod-Paul K, Gillen AE, Kopin EG, Gibney K, Elias AD, et al. Analysis of circulating breast cancer cell heterogeneity and interactions with peripheral blood mononuclear cells. Mol Carcinogenesis (2020) 59:112939. doi: $10.1002 / \mathrm{mc} .23242$

75. Cheng Y-H, Chen Y-C, Lin E, Brien R, Jung S, Chen Y-T, et al. Hydro-Seq enables contamination-free high-throughput single-cell RNA-sequencing for circulating tumor cells. Nat Commun (2019) 10(1):2163. doi: 10.1038/s41467019-10122-2
76. Pardoll DM. The blockade of immune checkpoints in cancer immunotherapy. Nat Rev Cancer (2012) 12:252-64. doi: 10.1038/nrc3239

77. Szakacs G, Paterson JK, Ludwig JA, Booth-Genthe C, Gottesman MM. Targeting multidrug resistance in cancer. Nat Rev Drug Discovery (2006) 5:219-34. doi: 10.1038/nrd1984

78. Perna D, Karreth FA, Rust AG, Perez-Mancera PA, Rashid M, Iorio F, et al. BRAF inhibitor resistance mediated by the AKT pathway in an oncogenic BRAF mouse melanoma model. Proc Natl Acad Sci U.S.A. (2015) 112:E53645. doi: $10.1073 /$ pnas.1418163112

79. Jerby-Arnon L, Shah P, Cuoco MS, Rodman C, Su MJ, Melms JC, et al. A Cancer Cell Program Promotes T Cell Exclusion and Resistance to Checkpoint Blockade. Cell (2018) 175:984-97 e924. doi: 10.1016/ j.cell.2018.09.006

80. Wu T, Wu X, Wang HY, Chen L. Immune contexture defined by single cell technology for prognosis prediction and immunotherapy guidance in cancer. Cancer Commun (Lond) (2019) 39:21. doi: 10.1186/s40880-019-0365-9

81. Chen Z, Ji Z, Ngiow SF, Manne S, Cai Z, Huang AC, et al. TCF-1-Centered Transcriptional Network Drives an Effector versus Exhausted CD8 T Cell-Fate Decision. Immunity (2019) 1(25):2343-7. doi: 10.1016/j.immuni.2019.09.013

82. Kini Bailur J, Mehta S, Zhang L, Neparidze N, Parker T, Bar N, et al. Changes in bone marrow innate lymphoid cell subsets in monoclonal gammopathy: target for IMiD therapy. Blood Adv (2017) 1:2343-7. doi: 10.1182/ bloodadvances.2017012732

83. Kubli SP, Vornholz L, Duncan G, Zhou W, Ramachandran P, Fortin J, et al. Fcmr regulates mononuclear phagocyte control of anti-tumor immunity. Nat Commun (2019) 10:2678. doi: 10.1038/s41467-019-10619-w

84. Hu Y, An Q, Sheu K, Trejo B, Fan S, Guo Y. Single Cell Multi-Omics Technology: Methodology and Application. Front Cell Dev Biol (2018) 6:28. doi: $10.3389 /$ fcell.2018.00028

85. Andor N, Lau BT, Catalanotti C, Kumar V, Sathe A, Belhocine K, et al. Joint single cell DNA-Seq and RNA-Seq of gastric cancer reveals subclonal signatures of genomic instability and gene expression. Biorxiv (2018). doi: $10.1101 / 445932$

86. Lubeck E, Coskun AF, Zhiyentayev T, Ahmad M, Cai L. Single-cell in situ RNA profiling by sequential hybridization. Nat Methods (2014) 11:360-1. doi: 10.1038/nmeth.2892

87. Chen KH, Boettiger AN, Moffitt JR, Wang S, Zhuang X. RNA imaging. Spatially resolved, highly multiplexed RNA profiling in single cells. Science (2015) 348:aaa6090. doi: 10.1126/science.aaa6090

88. Lee JH, Daugharthy ER, Scheiman J, Kalhor R, Ferrante TC, Terry R, et al. Fluorescent in situ sequencing (FISSEQ) of RNA for gene expression profiling in intact cells and tissues. Nat Protoc (2015) 10:442-58. doi: 10.1038/ nprot.2014.191

89. Lovatt D, Ruble BK, Lee J, Dueck H, Kim TK, Fisher S, et al. Transcriptome in vivo analysis (TIVA) of spatially defined single cells in live tissue. Nat Methods (2014) 11:190-6. doi: 10.1038/nmeth.2804

90. Hwang B, Lee JH, Bang D. Single-cell RNA sequencing technologies and bioinformatics pipelines. Exp Mol Med (2018) 50(8):96. doi: 10.1038/s12276018-0071-8

91. Aran D, Looney AP, Liu L, Wu E, Fong V, Hsu A, et al. Reference-based analysis of lung single-cell sequencing reveals a transitional profibrotic macrophage. Nat Immunol (2019) 20:163-72. doi: 10.1038/s41590-0180276-y

92. Trapnell C, Cacchiarelli D, Grimsby J, Pokharel P, Li S, Morse M, et al. The dynamics and regulators of cell fate decisions are revealed by pseudotemporal ordering of single cells. Nat Biotechnol (2014) 32:381-6. doi: 10.1038/nbt.2859

Conflict of Interest: The authors declare that the research was conducted in the absence of any commercial or financial relationships that could be construed as a potential conflict of interest.

Copyright (c) $2021 \mathrm{Liu}, \mathrm{Xu}$, Jin, Huang and Zhang. This is an open-access article distributed under the terms of the Creative Commons Attribution License (CC BY). The use, distribution or reproduction in other forums is permitted, provided the original author(s) and the copyright owner(s) are credited and that the original publication in this journal is cited, in accordance with accepted academic practice. No use, distribution or reproduction is permitted which does not comply with these terms. 